3. Schietinger $A$, et al. Tumor-specific $T$ cell dysfunction is a dynamic antigen-driven differentiation program initiated early during tumorigenesis. Immunity 2016;45:389-401. doi:10.1016/j.immuni.2016.07.011

4. Schietinger A, Greenberg PD. Tolerance and exhaustion: defining mechanisms of $T$ cell dysfunction. Trends Immunol 2014;35:51-60. doi:10.1016/j.it.2013.10.001

5. Ghoneim HE, et al. De novo epigenetic programs inhibit PD-1 blockade-mediated T cell rejuvenation. Cell 2017;170:142-157.e119. doi:10.1016/j.cell.2017.06.007

6. Pauken $\mathrm{KE}$, et al. Epigenetic stability of exhausted $\mathrm{T}$ cells limits durability of reinvigoration by PD-1 blockade. Science 2016;354:1160-1165. doi:10.1126/science. aaf2807

http://dx.doi.org/10.1136/jitc-2020-SITC2020.0769

\section{ANALYSIS OF GUT MICROBIOME IN PATIENTS RECEIVING ADOPTIVE T-CELL THERAPY (ACT) ACROSS DIFFERENT SOLID TUMOUR TYPES}

Arantzazu Barquin Garcia*, Sergio Ruiz, Paloma Navarro, Juan Francisco RodriguezMoreno, Elena Sevillano, Monica Yague, Sandra Amarilla, Jesus Garcia-Donas. Centro Integral Oncológico Clara Campal, Madrid, Spain

Background Tumour Infiltrating Lymphocytes (TILs) is a modality of ACT under development in solid tumours. Unfortunately, prior lymphodepletion is a key step that frequently requires the administration of antibiotics and antifungics for long periods of time. Although there is evidence that gut microbiome may influence tumour response in patients treated with checkpoint-inhibitors, it has not been extensively studied in ACT. $^{1}$

Methods Analysis of gut microbiome at three different times (T1: before lymphodepletion, T2: before TIL infusion and T3: day +15) has been performed in patients treated with ACT between 2018 and 2020. The composition and structure of the sampled microbial communities was assessed through the amplification and sequencing the V3-V4 variable regions of the $16 \mathrm{~S}$ rRNA gene. The Illumina Miseq sequencing $300 \times 2$ approach was used. Taxonomic assignment of phylotypes was performed using a Bayesian Classifier trained with Silva database version 132 (99\% OTUs full-length sequences). The following metrics were measured: observed OTUs (community richness), evenness (Pielou's index) and Shannon's diversity index. Differential abundance of taxa was tested using ANCOM test and Kruskal Wallis test.

Results A total of 21 patients have been treated with TILs between 2018 and 2020 at our institution. 67\% were female. Median age was 43 (range 26-70 years). All patients had stage IV pre-treated solid tumours: 55\% cervical cancer, 33\% melanoma, 10\% lung adenocarcinoma and 5\% head and neck cancer. Median previous treatment lines was 3 (range 2-4). Analysis of gut microbiome has been performed in 3 of these patients: one achieved PR, one progressed and the third one suffered an unexpected death. 971 phylotypes were detected. Analysis revealed differences in terms of observed OTUs, evenness and Shannon's index when comparing T1 and T2 with T3. At T3 a tendency towards less diversity and evenness was observed when compared with $\mathrm{T} 1$ and $\mathrm{T} 2(\mathrm{H} 3.0$, pvalue 0.083 , not statistically significant). Comparing the distribution of considered taxa in ACT responders vs. non-responders, we observed significant differences for both class (Bacteroidia, Clostridia and Gammaproteobacteria) and order (Bacteroidales, Lactobacillales, Clostridiales and Enterobacteriales) levels.

Conclusions A deep change in gut microbiome composition along TILs therapy was observed. Though preliminary, differences between responders and non-responders were observed but should be confirmed in larger populations.

\section{REFERENCE}

1. Gopalakrishnan V, Spencer CN, Nezi L, et al. Gut microbiome modulates response to anti-PD-1 immunotherapy in melanoma patients. Science 2018;359:97-103.

http://dx.doi.org/10.1136/jitc-2020-SITC2020.0770

\section{CHARACTERIZATION OF TUMOR INFILTRATING IMMUNE CELLS FROM ADULT SOFT TISSUE SARCOMAS}

'Jacky Chen*, ${ }^{2}$ Jay Wunder, ${ }^{1}$ Nalan Gokgoz, ${ }^{1}$ Irene Andrulis. 'Lunenfeld Tanenbaum Research Institute, Toronto, Canada; ${ }^{2}$ Sinai Health System, Toronto, Canada

Background Sarcoma is a group of rare bone and soft tissue tumors with over 50 distinct subtypes. Survival rate ranges widely due to the lack of efficacious treatments. Immunotherapy, such as adoptive cell therapy (ACT), has drawn great interest due to its minimal toxicity. In ACT, tumor infiltrating lymphocytes (TILs) are isolated from patients, expanded, and autologously reinfused back. We recently observed TIL's presence in Undifferentiated Pleomorphic Sarcoma (UPS) and Myxofibrosarcoma (MFS) tumors and found that tumor's PDL1 overexpression is correlated with better clinical outcome in UPS but not MFS. ${ }^{1}$ The Thelper1 inflammatory pathway was highly activated in the former subtype, which may explain the better outcome. These results illustrate the immunological differences where TILs may play a critical role. We hypothesize that there are phenotypic and functional differences between TILs of UPS and MFS that may be related to clinical outcomes. Sarcoma TILs are rare and challenging to culture

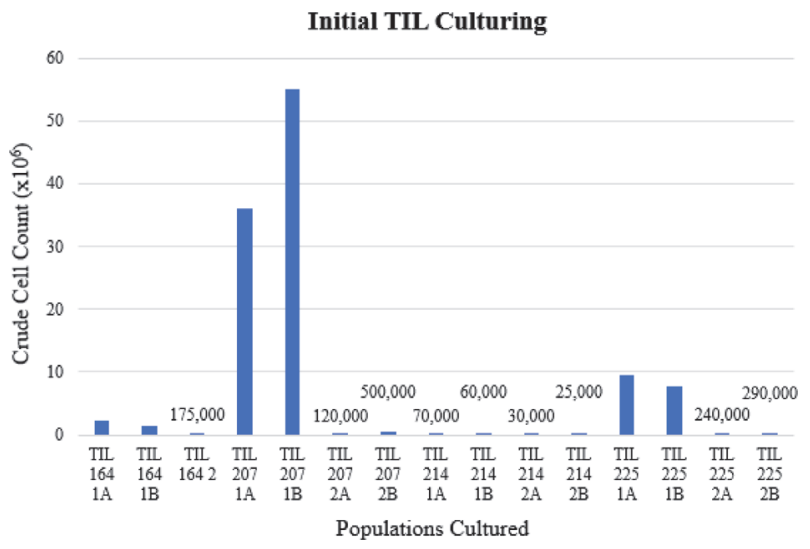

\section{Abstract 771 Figure 1}

Initial culturing of four primary MFS tumor cases with complete media (CM) over 4 weeks. Ten total cases were selected, five cases for each UPS and MFS sarcoma subtypes. To date, four MFS cases \#164, 207, 214 , and 225 have been processed. TIL populations were identified and categorized based on their growth rates and labelled as ' 1 ' or ' 2 ' representing 'fast' or 'slow' growing TILs, respectively. ' $A$ ' and ' $B$ ' represent technical replicates. Population TIL 164 ' 2 ' has no replicates. 15 populations were derived from the four MFS cases. TILs were cultured and expanded from tumor fragments in CM over 4 weeks in duration. CM consisted of Iscove's Modified Dulbecco's Medium, 6000 $\mathrm{IU} / \mathrm{mL} \mathrm{IL}-2,10 \%$ human serum albumin, $25 \mathrm{mmol} / \mathrm{L}$ HEPES, $2 \mathrm{mmol} \mathrm{L}-$ glutamine, $5.5 \times 10-5 \mathrm{~mol} / \mathrm{L} \beta$-mercaptoethanol, $100 \mathrm{U} / \mathrm{mL}$ penicillin, and $100 \mu \mathrm{g} / \mathrm{mL}$ streptomycin. At week 4, cells were collected and counted with a hemocytometer. Only 6 populations achieved $\geq 1 \times 106$ cells and are categorized as high initial cell count populations. 9 populations achieved $<1 \times 106$ cells and are categorized as low initial cell count populations. The 9 low initial cell count populations were further numbered with specific cell counts in figure 1 for clarity. These cell yields, with the exception of TIL $2071 \mathrm{~A}$ and 1B, are insufficient for characterization experiments. 